\title{
Study of the step structure of vicinal Si(110) surfaces using optical second harmonic generation
}

\author{
G.G. Malliaras ${ }^{1}$, H.A. Wierenga and Th. Rasing \\ Research Institute for Materials, University of Nijmegen, Toernooiveld, 6525 ED Nijmegen, Netherlands
}

Received 7 September 1992; accepted for publication 3 October 1992

Optical second harmonic generation studies of vicinal Si(110) show the possibility of measuring pure step contributions, thus creating an opportunity to determine the microscopic step structure at the silicon-silicon oxide interface.

\section{Introduction}

There has been considerable interest recently in the structure and dynamics of vicinal surfaces [1-6]. From a fundamental point of view, the thermodynamics of these surfaces form a challenging subject, including surface roughening and melting, step and kink formation and facetting. Vicinal surfaces are also very important for chemical reactivity (catalysis) and as substrates for epitaxial growth of crystals and nano-structures like quantum wires. A lot of experimental progress has been made using scanning tunneling microscopy [2,3], high resolution LEED [5] and transmission electron microscopy [6].

Recently, it was shown that optical second harmonic generation (SHG) has a remarkable sensitivity for the presence of steps on vicinal semiconductor [7-9] and metal [10] surfaces. Optical techniques have some definite advantages: they are applicable to any interface accessible by light, including those between dense media, while their high temporal resolution allows in situ monitoring of interface dynamics.

In this paper we will show how in certain cases, SHG can be used to derive microscopic information about the step structure, in particu-

\footnotetext{
I Present address: Polymer Chemistry, State University of Groningen, 9747 AG Groningen, Netherlands.
}

lar the presence and the density of kinks. This may lead to an intercsting new tool for in situ study of step dynamics.

\section{Theory}

SHG arises from the non-linear polarization $\boldsymbol{P}(2 \omega)$ induced by an incident laser field $\boldsymbol{E}(\omega)$. For centrosymmetric media, the surface allowed dipole term can be written as:

$P_{i}(2 \omega)=\chi_{i j k}^{(2)} E_{j}(\omega) E_{k}(\omega)$,

where $\chi^{(2)}$ is the second-order susceptibility tensor, reflecting the structure and symmetry properties of the surface layer.

In case of cubic media exited by a plane polarized field, higher-order (electric quadrupole and magnetic dipole) contributions to the second harmonic from the bulk can be ascribed to a polarization [11]:

$P_{i}(2 \omega)=\gamma \nabla_{i}[E(\omega) E(\omega)]+\zeta E_{i}(\omega) \nabla_{i} E_{i}(\omega)$,

where $\gamma$ and $\zeta$ are phenomenological constants giving the isotropic and anisotropic bulk contributions, respectively, and the $i$-directions are along the principal crystal axes.

For the case of a surface with $2 \mathrm{~m}$-symmetry, excited with $S$-polarized light, the S-polarized 
component of the radiated fields at $2 \omega$ is given by [12]:

$E_{\mathrm{s}, \mathrm{s}}(2 \omega)=C \zeta\left[\sin (2 \varphi)+\frac{3}{2} \sin (4 \varphi)\right]$,

where the proportionality constant $C$ contains the Fresnel factors. Eq. (3) shows that in case of $2 \mathrm{~m}$-symmetry the SHG only contains bulk contributions.

If the surface symmetry is reduced to $1 \mathrm{~m}$, surface terms appear that are given by:

$$
\begin{aligned}
E_{\mathrm{s}, \mathrm{s}}(2 \omega)= & C^{\prime}\left[\left(3 \chi_{y^{\prime} y y}+2 \chi_{x x y}+\chi_{y x x}\right) \cos (\varphi)\right. \\
& +\left(\chi_{y, y^{\prime}}-2 \chi_{x x y}-\chi_{y x x}\right) \\
& \times \cos (3 \varphi)]
\end{aligned}
$$

where the proportionally constant $C^{\prime}$ contains geometrical factors similar to eq. (3). Here, the approximation was made that the angle between the refracted beam and the surface normal is very small, which is justified by the large index of refraction of silicon.

\section{Experiment}

The samples were standard, optically flat, Syton-etched and polished silicon (110) wafers with a native oxide. The presence of the silicon oxide layer on the top of our samples does not complicate the analysis of our results since it is transparent and has a relatively low non-linearity $[13,14]$, thus giving a negligible contribution to the second harmonic signal.

Furthermore, it has been shown by HREM studies $[15,16]$ and photoemission experiments [17] that the boundary between several silicon crystal faces (e.g. $\mathrm{Si}(111), \mathrm{Si}(100)$ and $\mathrm{Si}(911)$ and silicon oxide is flat on an atomic scale (this was shown to be true for both native and thermal oxide [18]). We assume that this is also the case for the Si(110)-silicon oxide interface. Even if this assumption may not be totally justified, it will not affect the qualitative results of this work.

In fig. 1 the Si(110) surface is presented, where black and open circles represent $\mathrm{Si}$ atoms at different heights. Solid lines represent the inplane bonds, while dashed lines are the projec-

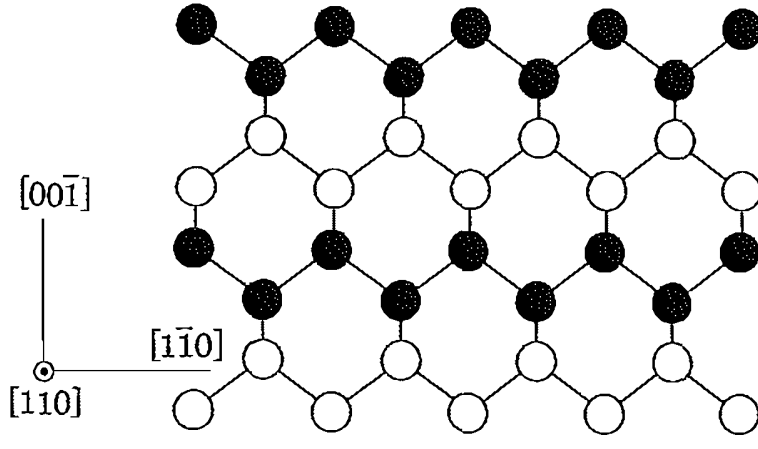

Fig. 1. The Si(110) surface, where black and open circles represent $\mathrm{Si}$ atoms at different heights (half a unit cell vector).

tions of the out-of-plane bonds. One observes a mirror plane parallel to the [001] axis and a glide plane (parallel to the (110) face). On a macroscopic level, an actual surface crystal layer will contain fractional amounts of both layers (the one with the black and the one with the white Si atoms), thus possessing an average $2 \mathrm{~m}$-symmetry.

The vicinal surface had a misorientation of $5^{\circ}$ towards [001], causing a step structure consisting of (110) terraces separated by steps perpendicular to the [001] direction. In the neighbourhood of the steps the macroscopic mirror symmetiy associated with the glide plane will be lost as a result of the symmetry breaking perpendicular to the step edge. On the other hand the mirror plane parallel to the [001] axis will not be affected.

For the SHG experiment we used the frequency doubled output at $532 \mathrm{~nm}$ of a Q-switched $\mathrm{Nd}$ :YAG laser, incident at an angle of $45^{\circ}$ on the sample. The fluence of the $8 \mathrm{~ns}$ pulses was limited to approximately $80 \mathrm{~mJ} / \mathrm{cm}^{2}$, well below the damage threshold and stable within $4 \%$. The reflected SHG intensity was recorded while the samples where stepwise rotated around their normal.

Two samples were studied, one precisely cut perpendicularly to the [110] direction and one cut with an offset angle of $5^{\circ}$ towards [001]. The orientation was checked by X-rays and the rotation angle $\varphi$ was defined as the angle between the plane of incindence and the [001] axis. 


\section{Results and discussion}

Measurements were performed for four polarization combinations for the incoming-outgoing light, namely $S_{\text {in }}-S_{\text {out }}, P_{\text {in }}-S_{\text {out }}, S_{\text {in }}-P_{\text {out }}$ and $P_{\text {in }}-$ $P_{\text {out }}$. They were all in perfect agreement with the existing phenomenological model [12] and the symmetry considerations outlined in the previous paragraph. In this paper we will only discuss the case of the $S_{\text {in }}-S_{\text {out }}$ polarization combination, as this one is the most sensitive to the step contributions. In fig. 2 we present the rotational anisotropy of both the $0^{\circ}$ and the $5^{\circ}$ sample. For the same input parameters, the difference in second harmonic intensity is striking: for $0^{\circ}$ offset the signal is two orders of magnitude smaller than for $5^{\circ}$

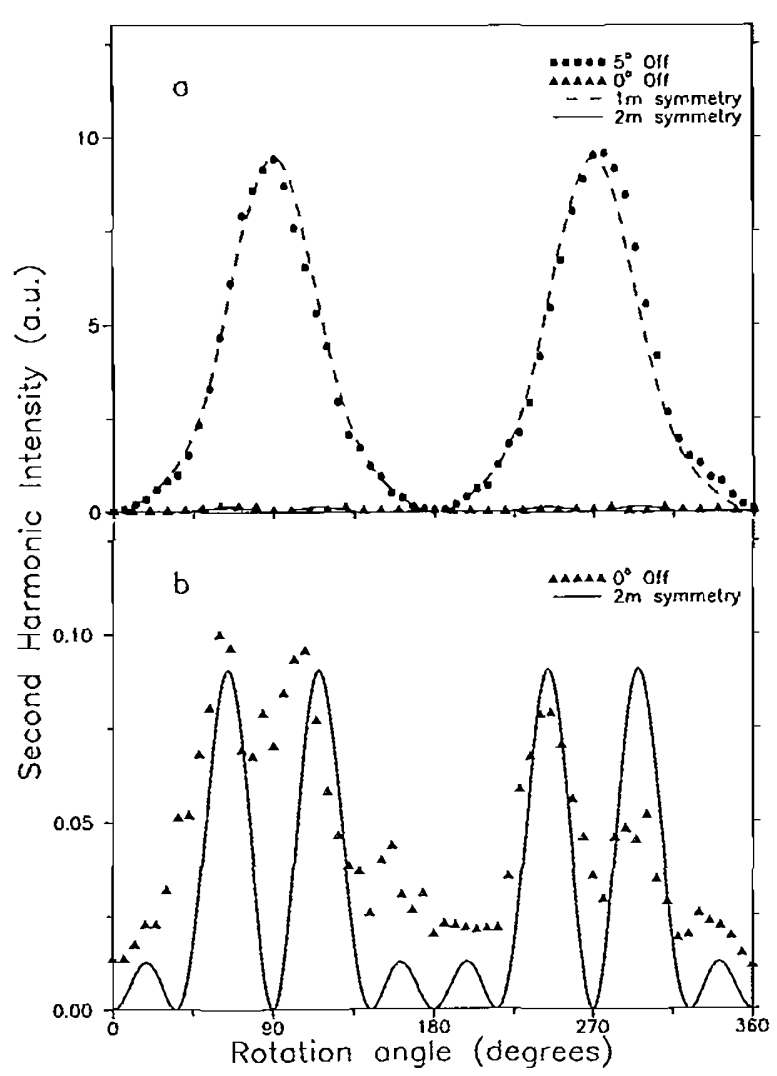

Fig. 2. Experimental results for the $S_{\text {in }}-S_{\text {out }}$ polarization combination: (a) sample with $5^{\circ}$ offset and sample with $0^{\circ}$ offset; (b) same sample with $0^{\circ}$ offet but with approximately 100 times increased sensitivity (see text for details). offset! The low signal from the $0^{\circ}$ sample shows that the higher-order bulk contributions are very weak at this polarization combination. The data are rather well fitted with eq. (3) (see fig. 2b).

The breaking of the $2 \mathrm{~m}$-symmetry at the steps of the misoriented sample results in a strong surface contribution, evident in fig. $2 \mathrm{a}$. The results from this vicinal surface reveal a $1 \mathrm{~m}$-symmetry, in accordance with the expectations mentioned above. The solid line is a fit using only eq. (4): the fact that the signal from the terraces and the bulk is negligible allows us to probe directly the steps! This also means that in this case we do not have any wobbling effects due to the fact that one looks under an offset angle at the Si(110) surface, as may be the case for the vicinal Si(111) [19].

It is well known that extra bonds appear on a stepped sample [20]. These are located at the step edges and will give a net contribution to the second harmonic signal. A microscopic model for the step contribution can be implemented, based on these extra step bonds.

In fig. 3 we propose a picture concerning these extra bonds. The $\mathrm{Si}-\mathrm{SiO}_{2}$ interface is assumed to be flat on an atomic scale, with the silica layer sitting on a perfect (110) layer of Si atoms. The step edges are running perpendicular to the [001] axis and they are interrupted by kinks. The extra $\mathrm{Si}-\mathrm{O}$ bonds are considered to be localized, with a $\delta$-function hyperpolarizability $\beta$ and an orientation defined by two angles, $\varphi_{1}$ and $\varphi_{2}, \varphi_{1}$ is the angle between the projection of the bond in the sample surface and the [001] axis, while $\varphi_{2}$ is the angle between the bond and the surface normal. It should be noted that there may still be dangling bonds present at these steps. This would give an additional set of parameters $\beta^{\prime}, \varphi_{1}^{\prime}$ and $\varphi_{2}^{\prime}$. Such an approach leads to an over-parameterizing of the system without yielding more information, as those cannot be determined independently. Therefore, we will ignore this possibility here.

We assume a truncated bulk and neglect small shifts in the orientation of the bonds due to interface strain [18]. The radiated fields from the extra step bonds can be easily calculated under the electric dipole approximation and for the case 
of the $S_{\mathrm{in}}-S_{\text {out }}$ polarization combination we find:

$$
\begin{aligned}
E_{\mathrm{s}, \mathrm{s}}(2 \omega) \sim \beta \sin ^{3}\left(\varphi_{2}\right)\left[\cos \left(\varphi_{1}\right) \cos (\varphi)\right. \\
\left.-\sin \left(\varphi_{1}\right) \sin (\varphi)\right]^{3}
\end{aligned}
$$

In the picture we show that the bonds located at the kinks have a different orientation than the bonds located at the straight step edges. On average the steps are straight, so kinks necessarily appear in pairs. This means that the number of kink bonds that have an angle $\varphi_{1}$ with the [001] axis equals the number of bonds having a $-\varphi_{1}$ angle. Macroscopically there will be a symmetric distribution with respect to the [001] axis and the

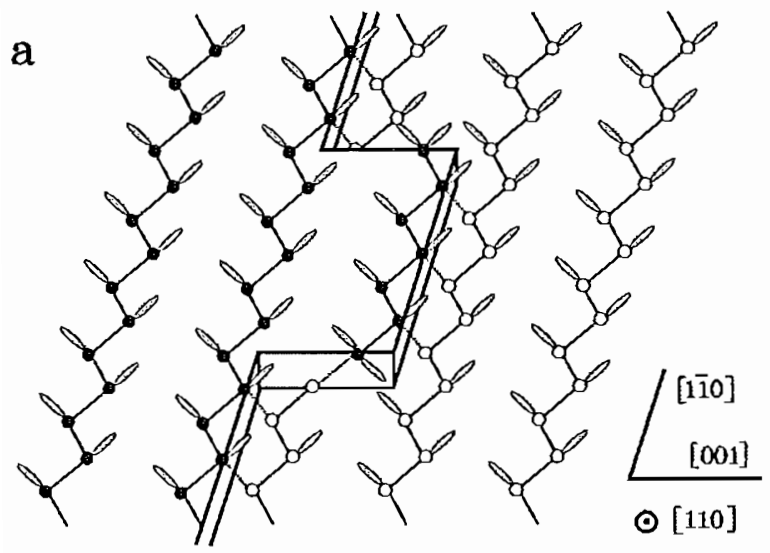

$\mathrm{b}$
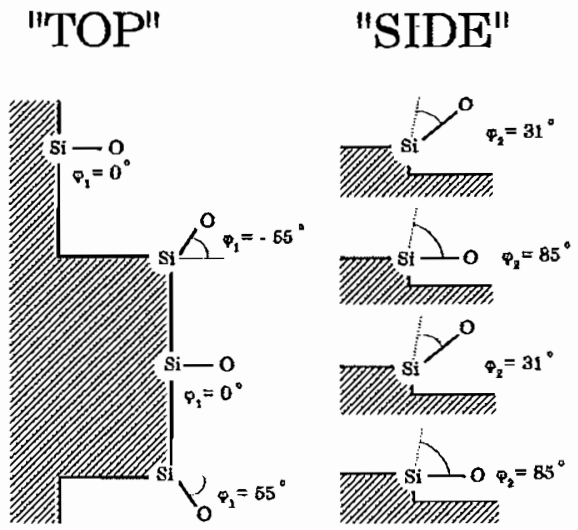

Fig. 3. (a) Three-dimensional view of a step, where black and open circles represent $\mathrm{Si}$ atoms at different heights (half a unit cell vector). Solid lines represent $\mathrm{Si}-\mathrm{Si}$ bonds perpendicular to [110], while dashed lines represent bonds between two $\mathrm{Si}$ atoms at difference heights. The $\mathrm{Si}-\mathrm{O}$ bonds are shown as lobes; (b) top and side view of the bonds located at steps and kinks, where the angles are like in the text.

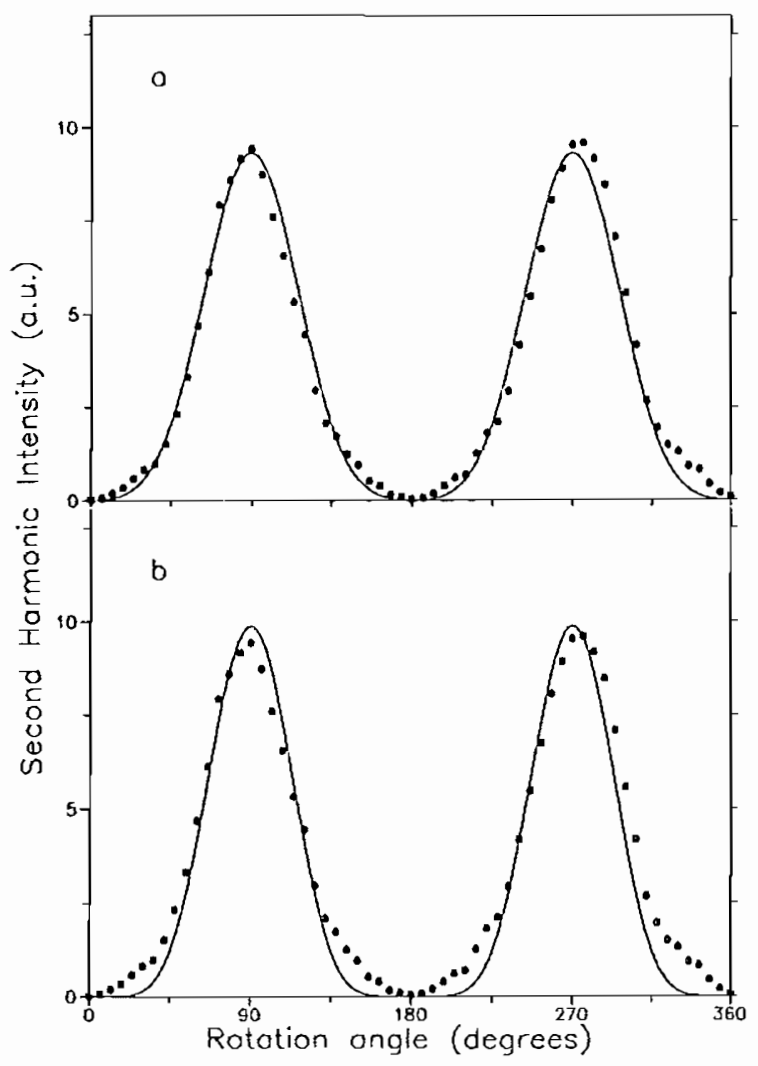

Fig. 4. Experimental results from the sample with $5^{\circ}$ offset: (a) the solid line is a fit to eq. (6), revealing an $x$ of $4.2 \%$; (b) for the sake of comparison, the solid line is a fit to eq. (6) with no kinks present $(x=0)$.

odd terms with respect to $\varphi_{1}$ of eq. (5) will vanish for the bonds located at the kinks. From the bulk structure, it follows that $\varphi_{1}=0^{\circ}$ and $\varphi_{2}=31^{\circ}$ for the straight step bonds and $\varphi_{1}=55^{\circ}$ and $\varphi_{2}=85^{\circ}$ for the kink bonds. The total contribution to the second harmonic generation from all the step bonds will then be:

$$
\begin{aligned}
E_{\mathrm{s}, \mathrm{s}}(2 \omega) \sim & \beta[(17.117 x+4.01) \cos (\varphi) \\
& -(9.515 x-1.337) \cos (3 \varphi)],
\end{aligned}
$$

where $x$ is the fraction of bonds that is located at the kinks.

Eq. (6) has the same dependence on the rotation angle as eq. (4), that was derived from macroscopic considerations. By means of the microscopic approach the two prefactors of eq. (4) have been interrelated. 
From a fit of this equation to the experimental data the fraction $x$ of the bonds located at the kinks, and thus the density of the kinks on the steps, can be deduced. In fig. 4a the fit of eq. (6) to the experimental data is presented, revealing an $x$ equal to $4.2 \% \pm 0.4 \%$. This value seems reasonable, meaning an average distance of approximately 100 ångström between adjacent kinks. For comparison, fig. $4 \mathrm{~b}$ shows a fit to eq. (6) with $x=0$, i.e. the case that no kinks are present.

Because the epitaxial growth of a film on a stepped surface is enhanced near the steps, the ideas that were outlined here can help to monitor and understand the first stages of epitaxial growth. The same holds for the study of surface roughening and melting, that will become apparent as an increase in the density of the kinks.

In conclusion we can say that we have taken advantage of the fact that the second harmonic signal from the $\mathrm{Si}(110)$ terraces and the bulk is almost negligible to develop a simple microscopic model for the contribution of the steps. By means of this approach, information can be retrieved in a straightforward way about the features of the steps. This, together with the capability of SHG for in situ measurements and its high temporal resolution, brings out a unique tool to study both step and kink density during roughening or growth.

\section{Acknowledgements}

Part of this work was supported by the Stichting voor Fundamenteel Onderzoek der Materie (FOM). We thank Professor T. Ichinokawa of the Waseda University in Japan for sending us the high quality flat $\mathrm{Si}(110)$ wafer. G.G.M. is grateful to the Foundation for Research and Technology
Hellas (FORTH) and thanks Professor K. Kambas of the Aristotle University of Thessaloniki for fruitful discussions and valuable suggestions.

\section{References}

[1] E. Pehlke and J. Tersoff, Phys. Rev. Lett. 67 (1991) 1290.

[2] B.S. Swartzentruber, Y.W. Mo, R. Kariotis, M.G. Lagally and M.B. Webb, Phys. Rev. Lett. 65 (1990) 1913.

[3] X.S. Wang, J.L. Goldberg, N.C. Bartelt, T.L. Einstein and E.D. IVillians, Phys. Rev. Lett. 65 (1990) 2430.

[4] D.E. Wolf, Phys. Rev. Lett. 67 (1991) 1783.

[5] R.J. Phaneuf, N.C. Bartelt, E.D. Willitms, W. Swiech and E. Bauer, Phys. Rev. Lett. 67 (1991) 2986.

[6] F.M. Ross and J.M. Gibson, Phys. Rev. Lett. 68 (1992) 1782.

[7] C.W. van Hasselt, M.A. Verheijen and Th. Rasing, Phys. Rev, B 42 (1990) 9263.

[8] M.A. Verheijen, C.W. van Hasselt and Th. Rasing, Surf. Sci. 251 (1991) 471.

[9] R.W.J. Hollering, D. Dijkkamp and H.B. Elswijk, Surf. Sci. 243 (1991) 121.

[10] S. Janz, D.J. Bottomley, H.M. van Driel and R.S. Timsit, Phys. Rev. Lett. 66 (1991) 1201.

[11] N. Bloembergen, R.K. Chang, S.S. Jha and C.H. Lee, Phys. Rev. 174 (1968) 813.

[12] J.E. Sipe, D.J. Moss and H.M. van Driel, Phys. Rev. B 35 (1987) 1129.

[13] H.W.K. Tom, T.F. Heinz and Y.R. Shen, Phys. Rev. Lett. 51 (1983) 1983.

[14] Molecular Structure and Properties, Phys. Chem. Ser. 2, Vol. 2, Ed. A.D. Buckingham (Buttersworth, London, 1975) ch. 5.

[15] O.L. Krivanek and J.H. Mazur, Appl. Phys. Lett. 37 (1980) 392.

[16] O.L. Krivanek, D.C. Tsui, T.T. Sheng and A. Kamgar, in: Properties of $\mathrm{SiO}_{2}$ and its Interfaces, Ed. S.T., Pantelides (Pergamon, New York, 1978).

[17] T.H. DiStefano, J. Vac. Sci. Technol. 13 (1976) 856.

[18] A. Ourmazd, D.W. Taylor, J.A. Rentschler and J. Bevk, Phys. Rev. Lett. 59 (1987) 213.

[19] R.W. Hollering and M. Barmentlo, Opt. Commun. 8 (1992) 141.

[20] B.Z. Olshanetsky and A.A. Shklyaev, Surf. Sci. 82 (1979) 445. 